\title{
Erratum to: Data and Information Quality: Dimensions, Principles and Techniques
}

\author{
Carlo Batini and Monica Scannapieco
}

\section{Erratum to:}

C. Batini, M. Scannapieco, Data and Information Quality, Data-Centric Systems and Applications, DOI 10.1007/978-3-319-24106-7

The affiliation of the author Monica Scannapieco has been incorrectly captured in Page IV and the correct affiliation is as follows:

Istituto Nazionale di Statistica-Istat, Rome, Italy.

The names of co-authors have been missed in the Chapter opening pages of Chapters 4 (page 87), 5 (page 113), 13 (page 403) and 14 (page 421). The correct information is as follows:

Chapter 4 has been written by Anisa Rula, Andrea Maurino, and Carlo Batini.

Chapter 5 has been written by Gianluigi Ciocca, Silvia Corchs, Francesca Gasparini, Carlo Batini, and Raimondo Schettini.

Chapter 13 has been written by Federico Cabitza and Carlo Batini.

Chapter 14 has been written by Monica Scannapieco and Laure Berti.

The online version of the updated book can be found under

DOI 10.1007/978-3-319-24106-7 